\title{
Machine Learning in Manufacturing: Review, Synthesis, and Theoretical Framework
}

\section{Ajit Sharma(*), Zhibo Zhang(^), Rahul Rai(\#)}

(^) Manufacturing and Design Lab (MADLab), University of Buffallo, Buffalo, NY, USA.

(\#) Geometric Reasoning and Artifiicial Intelligence Lab (GRAIL), Clemson University, Greenville, SC, USA.

Complete List of $\mathbf{2 5 0}$ Articles Subjected to Descriptive Literature Review

\begin{tabular}{|c|c|c|c|}
\hline ID & YEAR & AUTHOR & TITLE \\
\hline & 2019 & Li, Zhixiong; Zhang, Ziyang; Shi, Junchuan; Wu, Dazhong; & Prediction of surface roughness in extrusion-based additive manufacturing with machine learning \\
\hline 2 & 2019 & Kumar, Akhilesh; Chinnam, Ratna Babu; Tseng, Finn; & An HMM and polynomial regression based approach for remaining useful life and health state estimation of cutting tools \\
\hline 3 & 2019 & Boudjelida, Abdelhamid; & On the robustness of joint production and maintenance scheduling in presence of uncertainties \\
\hline 4 & 2019 & Aminzadeh, Masoumeh; Kurfess, Thomas R; & Online quality inspection using Bayesian classification in powder-bed additive manufacturing from high-resolution visual camera images \\
\hline 5 & 2019 & Liu, Lizhe and Cao, Danhua and Wu, Yubin and Wei, Taoran & Defective samples simulation through adversarial training for automatic surface inspection \\
\hline 6 & 2019 & Zhang, Binbin and Jaiswal, Prakhar and Rai, Rahul and Guerrier, Paul and Baggs, George & Convolutional neural network-based inspection of metal additive manufacturing parts \\
\hline 7 & 2018 & Jurkovic, Zoran; Cukor, Goran; Brezocnik, Miran; Brajkovic, Tomislav; & A comparison of machine learning methods for cutting parameters prediction in high speed turning process \\
\hline 8 & 2018 & Wu, Yuting; Yuan, Mei; Dong, Shaopeng; Lin, Li; Liu, Yingqi; & Remaining useful life estimation of engineered systems using vanilla LSTM neural networks \\
\hline & 2018 & Khakifirooz, Marzieh; Chien, Chen Fu; Chen, Ying-Jen; & Bayesian inference for mining semiconductor manufacturing big data for yield enhancement and smart production to empower industry 4.0 \\
\hline 10 & 2018 & Ragab, Ahmed; El-Koujok, Mohamed; Poulin, Bruno; Amazouz, Mouloud; Yacout, Soumaya; & Fault diagnosis in industrial chemical processes using interpretable patterns based on Logical Analysis of Data \\
\hline 11 & 2018 & Ruiz, Magda; Mujica, Luis E; Alferez, Santiago; Acho, Leonardo; Tutiven, Christian; Vidal, Yolanda; Rodellar, Jose; Pozo, Francesc; & Wind turbine fault detection and classification by means of image texture analysis \\
\hline 12 & 2018 & Liu, Changqing; Li, Yingguang; Zhou, Guanyan; Shen, Weiming; & A sensor fusion and support vector machine based approach for recognition of complex machining conditions \\
\hline 13 & 2018 & Diez-Olivan, Alberto; Pagan, Jose A; Khoa, Nguyen Lu Dang; Sanz, Ricardo; Sierra, Basilio; & Kernel-based support vector machines for automated health status assessment in monitoring sensor data \\
\hline 14 & 2018 & Kumar, Ajay; Shankar, Ravi; Thakur, Lakshman S; & A big data driven sustainable manufacturing framework for condition-based maintenance prediction \\
\hline$\frac{17}{15}$ & 2018 & Animah, Isaac; Shafiee, Mahmood; & Condition assessment, remaining useful life prediction and life extension decision making for offshore oil and gas assets \\
\hline 16 & 2018 & Liu, Zheng; Meyendorf, Norbert; Mrad, Nezih; & The role of data fusion in predictive maintenance using digital twin \\
\hline 17 & 2018 & Cui, Weiwei; Lu, Zhiqiang; Li, Chen; Han, Xiaole; & A proactive approach to solve integrated production scheduling and maintenance planning problem in flow shops \\
\hline 18 & 2018 & McComb, Christopher; Meisel, Nicholas; Murphy, Christian; Simpson, TW; & Predicting Part Mass, Required Support Material, and Build Time via Autoencoded Voxel Patterns \\
\hline 19 & 2018 & Chan, Siu L; Lu, Yanglong; Wang, Yan; & Data-driven cost estimation for additive manufacturing in cybermanufacturing \\
\hline 20 & 2018 & Zhu, Zuowei; Anwer, Nabil; Huang, Qiang; Mathieu, Luc; & Machine learning in tolerancing for additive manufacturing \\
\hline 21 & 2018 & Yang, Zhuo; Eddy, Douglas; Krishnamurty, Sundar; Grosse, Ian; Denno, Peter;, Witherell, Paul William; Lopez, Felipe; & Dynamic metamodeling for predictive analytics in advanced manufacturing \\
\hline 22 & 2018 & Yan, Wentao; Lin, Stephen; Kafka, Orion L; Lian, Yanping; Yu, Cheng; Liu, Zeliang; Yan, Jinhui; Wolff, Sarah; Wu, Hao; Ndip-Agbor, Ebot; & Data-driven multi-scale multi-physics models to derive process-structure-property relationships for additive manufacturing \\
\hline 23 & 2018 & Kappes, Branden; Moorthy, Senthamilaruvi; Drake, Dana; Geerlings, Henry; Stebner, Aaron; & Machine learning to optimize additive manufacturing parameters for laser powder bed fusion of Inconel 718 \\
\hline 24 & 2018 & Yang, Zhuo; Eddy, Douglas; Krishnamurty, Sundar; Grosse, Ian; Lu, Yan; & A Super-Metamodeling Framework to Optimize System Predictability \\
\hline 25 & 2018 & Imani, Farhad; Gaikwad, Aniruddha; Montazeri, Mohammad; Rao, Prahalada; Yang, Hui; Reutzel, Edward; & Layerwise in-process quality monitoring in laser powder bed fusion \\
\hline 26 & 2018 & Scime, Luke; Beuth, Jack; & Anomaly detection and classification in a laser powder bed additive manufacturing process using a trained computer vision algorithm \\
\hline 27 & 2018 & Scime, Luke; Beuth, Jack; & A multi-scale convolutional neural network for autonomous anomaly detection and classification in a laser powder bed fusion additive manufacturing process \\
\hline 28 & 2018 & Zhang, Yingiji; Hong, Geok Soon; Ye, Dongsen; Zhu, Kunpeng; Fuh, Jerry YH; & Extraction and evaluation of melt pool, plume and spatter information for powder-bed fusion AM process monitoring \\
\hline 29 & 2018 & Shevchik, Sergey A; Kenel, Christoph; Leinenbach, Christian; Wasmer, Kilian; & Acoustic emission for in situ quality monitoring in additive manufacturing using spectral convolutional neural networks \\
\hline 30 & 2018 & Ye, Dongsen; Hong, Geok Soon; Zhang, Yingiie; Zhu, Kunpeng; Fuh, Jerry Ying Hsi; & Defect detection in selective laser melting technology by acoustic signals with deep belief networks \\
\hline 31 & 2018 & Grasso, Marco; Gallina, Francesco; Colosimo, Bianca Maria; & Data fusion methods for statisistical process monitoring and quality characterization in metal additive manufacturing \\
\hline 32 & 2018 & Wang, Zirui and Wang, Jun and Wang, Youren & $\mathrm{An}$ intelligent diagnosis scheme based on generative adversarial learning deep neural networks and its application to planetary gearbox fault pattern recognition \\
\hline 33 & 2018 & Yao, Bing; Imani, Farhad; Yang, Hui; & Markov decision process for image-guided additive manufacturing \\
\hline 34 & 2017 & Garcia-Ordás, Maite; & Wear characterization of the cutting tool in milling processess using shape and texture descriptors \\
\hline 35 & 2017 & Wu, Dazhong; Jennings, Connor; Terpenny, Janis; Gao, Robert X; Kumara, Soundar; & A comparative study on machine learning algorithms for smart manufacturing: tool wear prediction using random forests \\
\hline 36 & 2017 & Arnold, F; Hänel, A; Nestler, A; Brosius, A; & New Approaches for the Determination of Specific Values for Process Models in Machining Using Artificial Neural Networks \\
\hline 37 & 2017 & D'Addona, Doriana M; Ullah, AMM Sharif; Matarazzo, D; & Tool-wear prediction and pattern-recognition using artificial neural network and DNA-based computing \\
\hline 38 & 2017 & Saravanamurugan, S; Thiyagu, S; Sakthivel, NR; Nair, Binoy B; & Chatter prediction in boring process using machine learning technique \\
\hline 39 & 2017 & Ren, Ruoxu; Hung, Terence; Tan, Kay Chen; & A generic deep-learning-based approach for automated surface inspection \\
\hline 40 & 2017 & Lu, Chen; Wang, Zhenya; Zhou, Bo; & Intelligent fault diagnosis of rolling bearing using hierarchical convolutional network based health state classification \\
\hline$\frac{71}{41}$ & 2017 & Verstraete, David; Ferrada, Andrés; Droguett, Enrique López; Meruane, Viviana; Modarres, Mohammad; & Deep learning enabled fault diagnosis using time-frequency image analysis of rolling element bearings \\
\hline 42 & 2017 & Wang, Peng; Yan, Ruqiang; Gao, Robert X; & Virtualization and deep recognition for system fault classification \\
\hline 43 & 2017 & Lu, Chen; Wang, Zhen-Ya; Qin, Wei-Li; Ma, Jian; & Fault diagnosis of rotary machinery components using a stacked denoising autoencoder-based health state identification \\
\hline 44 & 2017 & Shao, Haidong; Jiang, Hongkai; Wang, Fuan; Zhao, Huiwei; & An enhancement deep feature fusion method for rotating machinery fault diagnosis \\
\hline 45 & 2017 & Guo, Xiaojie; Shen, Changqing; Chen, Liang; & Deep fault recognizer: An integrated model to denoise and extract features for fault diagnosis in rotating machinery \\
\hline 46 & 2017 & Chen, Zhiqiang; Deng, Shengcai; Chen, Xudong; Li, Chuan; Sanchez, Rene-Vinicio; Qin, Huafeng; & Deep neural networks-based rolling bearing fault diagnosis \\
\hline 47 & 2017 & Zhao, Rui; Wang, Dongzhe; Yan, Ruqiang; Mao, Kezhi; Shen, Fei; Wang, Jinjiang; & Machine health monitoring using local feature-based gated recurrent unit networks \\
\hline 48 & 2017 & Zhao, Rui; Yan, Ruqiang; Wang, Jinjiang; Mao, Kezhi; & Learning to monitor machine health with convolutional bi-directional LSTM networks \\
\hline 49 & 2017 & Wang, Peng; Gao, Robert X; Yan, Ruqiang; & A deep learning-based approach to material removal rate prediction in polishing \\
\hline 50 & 2017 & Deutsch, Jason; He, Miao; He, David; & Remaining useful life prediction of hybrid ceramic bearings using an integrated deep learning and particle filter approach \\
\hline 51 & 2017 & Chien, Chen-Fu; Liu, Chiao-Wen; Chuang, Shih-Chung; & Analysing semiconductor manufacturing big data for root cause detection of excursion for yield enhancement \\
\hline 52 & 2017 & Franciosa, Pasquale; Palit, Arrab; Vitolo, Ferdinando; Ceglarek, Darek; & Rapid response diagnosis of multi-stage assembly process with compliant non-ideal parts using self-evolving measurement system \\
\hline 53 & 2017 & Chen, Hongge; Boning, Duane; & Online and incremental machine learning approaches for IC yield improvement \\
\hline 54 & 2017 & Jian, Chuanxia; Gao, Jian; Ao, Yinhui; & Automatic surface defect detection for mobile phone screen glass based on machine vision \\
\hline 55 & 2017 & Manco, Giuseppe; Ritacco, Ettore; Rullo, Pasquale; Gallucci, Lorenzo; Astill, Will; Kimber, Dianne; Antonelli, Marco; & Fault detection and explanation through big data analysis on sensor streams \\
\hline 56 & 2017 & Krishnakumari, A; Elayaperumal, A; Saravanan, M; Arvindan, C; & Fault diagnostics of spur gear using decision tree and fuzzy classifier \\
\hline
\end{tabular}




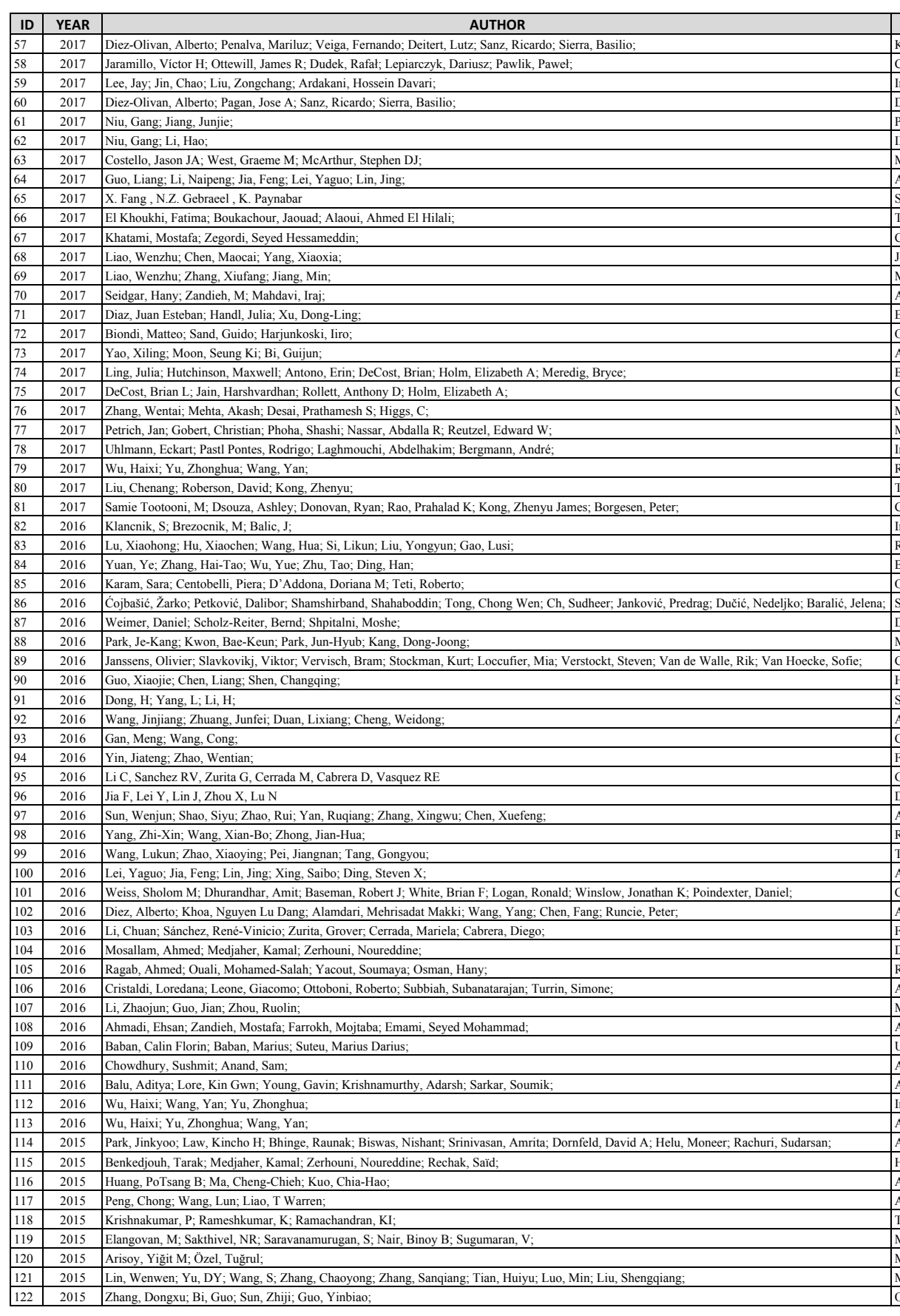

ernel density-based pattern classification in blind fasteners installation

TITLE

thestage Bayesian interence data fus Atroduction to data-driven methodologies for prognostics and health management Data-driven prognostics using a combination of constrained K-means clustering, fuzzy modeling and LOF-based score

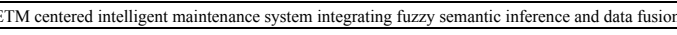
Machine learning model for event-based prognostics in gas circulator condition monitoring Scalable prognostic models for large-scale condition monitoring applications

The "Dual-Ants Colony": A novel hybrid approach for the flexible job shop scheduling problem with preventive maintenance cordinative production and maintenance scheduling problem with flexible maintenance time intervals bint optimization of preventive maintenance and production scheduling for parallel machines system Multi-objective group scheduling optimization integrated with preventive maintenance An efficient meta-heuristic algorithm for scheduling a two-stage assembly flow shop problem with preventive maintenance activities and reliability approach Evolutionary robust optimization in production planning-interactions between number of objectives, sample size and choice of robustness measure ptimization of multipurpose process plant operations: A multi-time-scale maintenance and production scheduling approach Milding Computer vision and machine learning for autonomous characterization of am powder feedstock Machine learning enabled powder spreading process map for metal additive manufacturing (AM) Machine Learning for Defect Detection for PBFAM Using High Resolution Layerwise Imaging Coupled with Post-Build CT Scans Real-time FDM machine condition monitoring and diagnosis based on acoustic emission and hidden semi-Markov model lassifying the dimensional variation in additive manufactured parts from laser-scanned three-dimensional point cloud data using machine learning approaches Research on the prediction model of micro-milling surface roughness of Inconel 718 based on SVM Bayesian learning-based model-predictive vibration control for thin-walled workpice machining processes Online prediction of cutting tool life in turning via cognitive decision making

Surface roughness prediction by extreme learning machine constructed with abrasive water jet achine learning-based imaging system for surface defect inspection

Convolutional neural network based fault detection for rotating machinery

lierarchical adaptive deep convolution neural network and its application to bearing fault diagnosis mall fault diagnosis of front-end speed controlled wind generator based

Construction of hierarchical diagnosis network based on deep learning and its application in the fault pattern recognition of rolling element bearings Fault diagnosis network design for vehicle on-board equipments of high-speed railway: A deep learning approach

Cearbox fault diagnosis based on deep random forest fusion of acoustic and vibratory signals eep neural networks: a promising tool for fault characteristic mining and intelligent diagnosis of rotating machinery with massive data sparse auto-encoder-based deep neural network approach for induction motor faults classification Representational learning for fault diagnosis of wind turbine equipment: A multi-layered extreme learning machines approach A C Continuous prediction of manufacturing performance throughout

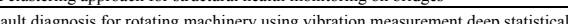
Data-driven prognostic method based on Bayesian approaches for direct remaining useful life prediction

emaining useful life prediction using prognostic methodology based on logical analysis of data and Kaplan-Meier estimation A comparative study on data-driven prognostic approaches using fleet knowledge mization approach for flexible job shop scheduling problem under random machine breakdown by evolutionary algorithms sing a fuzzy logic approach for the predictive maintenance of textile machines Artificial neural network based geometric compensation for thermal deformation in additive manufacturing processes deep 3d convolutional neural network based design for manufacturability framework In situ monitoring of FDM machine condition via acoustic emission appoach for online monitoring of additive manufacturing based on acoustic emission A ger A APNN self-learning tool breakage detection system in end milling operations new method for the prediction of chater stability lobes based on dynamic cutting force simulation model and support vector machine Mal wear condition prediction using vibration signals in high speed machining (HSM) of titanium (Ti-6Al-4 V) alloy Machine learning based predictive modeling of machining induced microhardness and grain size in $\mathrm{Ti}_{\mathrm{i}}-\mathrm{Al}-\mathrm{Al}$ - $\mathrm{V}$ alloy $\mathrm{al}$ turning Multi-objective teaching-learning-based optimization algorithm for reducing carbon emissions and operation time in turning operations Onltine monitoring of preceision optics grinding using acoustic emission based on support vector machine 


\begin{tabular}{|c|c|c|}
\hline ID & YEAR & AUTHOR \\
\hline 123 & 2015 & Shaban, Yasser; Yacout, Soumaya; Balazinski, Marek; Meshreki, Mouhab; Attia, Helmi; \\
\hline 124 & 2015 & Teixidor, Daniel;; Grzenda, Maciej; Bustillo, Andrés; Ciurana, Joaquim; \\
\hline 125 & 2015 & Majumder, Arindam; \\
\hline 126 & 2015 & Chen, ZhiQiang; Li, Chuan; Sanchez, René-Vinicio; \\
\hline 127 & 2015 & Yu, Hongyang; Khan, Faisal; Garaniya, Vikram; \\
\hline 128 & 2015 & Shao, Haidong; Jiang, Hongkai; Zhang, Xun; Niu, Maogui; \\
\hline 129 & 2015 & Xie H, Yang Y, Wang $\mathrm{H}$, Li T, Jin $\mathrm{W}$ \\
\hline 130 & 2015 & Li C, Sanchez RV, Zutita G, Cerrada M, Cabrera D, Vasquez RE \\
\hline 131 & 2015 & Malhotra, Pankaj; Vig, Lovekesh; Shroff, Gautam; Agarwal, Puneet; \\
\hline 132 & 2015 & Diao, Guangzhou; Zhao, Liping; Yao, Yiyong; \\
\hline 133 & 2015 & Basurko, Oihane C; Uriondo, Zigor; \\
\hline 134 & 2015 & Baqqar, Mabrouka; \\
\hline 135 & 2015 & Garcia-Santiago, CA; Del Ser, Javier; Upton, C; Quilligan, F; Gil-Lopez, S; Salcedo-Sanz, Sancho; \\
\hline 136 & 2015 & Wang, Shijin; Liu, Ming; \\
\hline 137 & 2015 & Gaynor, Andrew Thomas; \\
\hline 138 & 2015 & Rao, Prahalad K; Liu, Jia Peter; Roberson, David; Kong, Zhenyu James; Williams, Christopher; \\
\hline 139 & 2014 & Bhinge, Raunak; Biswas, Nishant; Dornfeld, David; Park, Jinkyoo; Law, Kincho H; Helu, Moneer; Rachuri, Sudarsan; \\
\hline 140 & 2014 & Painuli, Sanidhya; Elangovan, M; Sugumaran, V; \\
\hline 141 & 2014 & Park, Hong-Seok; Tran, Ngoc-Hien; \\
\hline 142 & 2014 & AlThobiani, Faisal; Ball, Andrew; \\
\hline 143 & 2014 & Guo, Junqi; Xie, Xiaobo; Bie, Rongfang; Sun, Limin; \\
\hline 144 & 2014 & Qiu, Xueheng; Zhang, Le; Ren, Ye; Suganthan, Ponnuthurai N; Amaratunga, Gehan; \\
\hline 145 & 2014 & Chien, Chen-Fu; Chuang, Shih-Chung; \\
\hline 146 & 2014 & Yun, Jong Pil; Choi, Doo-chul; Jeon, Yong-ju; Park, Changhyun; Kim, Sang Woo; \\
\hline 147 & 2014 & Luo, Weiqiang; Rojas, Juan; Guan, TianQiang; Harada, Kensuke; Nagata, Kazuyuki; \\
\hline 148 & 2014 & Kirby E, Zhang Z, Chen JC \\
\hline 149 & 2014 & Safizadeh, MS; Latifi, SK; \\
\hline 150 & 2014 & Fathi, Alireza; Mozaffari, Ahmad; \\
\hline 151 & 2014 & Garg, Akhil; Tai, K; \\
\hline 152 & 2013 & Azadeh, Ali; Saberi, Morteza; Kazem, Ahmad; Ebrahimipour, Vahid; Nourmohammadzadeh, A; Saberi, Zahra; \\
\hline 153 & 2013 & Miao, En-Ming; Gong, Ya-Yun; Niu, Peng-Cheng; Ji, Chang-Zhu; Chen, Hai-Dong; \\
\hline 154 & 2013 & Deris, Ashanira Mat; Zain, Azlan Mohd; Sallehuddin, Roselina; \\
\hline 155 & 2013 & Tamilselvan, Prasanna; Wang, Pingfeng; \\
\hline 156 & 2013 & Chien, Chen-Fu; Hsu, Chia-Yu; Chen, Pei-Nong; \\
\hline 157 & 2013 & Lieber, Daniel; Stolpe, Marco; Konrad, Benedikt; Deuse, Jochen; Morik, Katharina; \\
\hline 158 & 2013 & Konrad, Benedikt; Lieber, Daniel; Deuse, Jochen; \\
\hline 159 & 2013 & Arif, Fahmi; Suryana, Nanna; Hussin, Burairah; \\
\hline 160 & 2013 & Weiss, Sholom M; Dhurandhar, Amit; Baseman, Robert J; \\
\hline 161 & 2012 & Masci, Jonathan; Meier, Ueli; Ciresan, Dan; Schmidhuber, Jürgen; Fricout, Gabriel; \\
\hline 162 & 2012 & Scholz-Reiter, Bernd; Weimer, Daniel; Thamer, Hendrik; \\
\hline 163 & 2012 & Ceglarek, D; Prakash, PKS; \\
\hline 164 & 2011 & Rao, RV; Kalyankar, VD; \\
\hline 165 & 2011 & Malhi, Arnaz; Yan, Ruqiang; Gao, Robert X; \\
\hline 166 & 2011 & Kumar, Naveen; Mastrangelo, Christina; Montgomery, Doug; \\
\hline 167 & 2011 & Chen, Ssu-Han; Perng, Der-Baau; \\
\hline 168 & 2011 & Perng, Der-Baau; Chen, Ssu-Han; \\
\hline 169 & 2011 & Palani, S; Natarajan, U; \\
\hline 170 & 2011 & Di Angelo, Luca; Di Stefano, Paolo; \\
\hline 171 & 2010 & Salahshoor, Karim; Kordestani, Mojtaba; Khoshro, Majid S; \\
\hline 172 & 2010 & Somashekhar, KP; Ramachandran, N; Mathew, Jose; \\
\hline 173 & 2010 & Rodriguez, Alberto; Bourne, David; Mason, Mathew; Rossano, Gregory F; Wang, JianJun; \\
\hline 174 & 2010 & Saravanan, N; Ramachandran, KI; \\
\hline 175 & 2010 & Dhanasekar, B; Ramamoorthy, B; \\
\hline 176 & 2010 & Lu, ZL; Li, DC; Lu, BH; Zhang, AF; Zhu, GX; Pi, G; \\
\hline 177 & 2009 & Kang, Pilsung; Lee, Hyoung-joo; Cho, Sungzoon; Kim, Dongili; Park, Jinwoo; Park, Chan-Kyoo; Doh, Seungyong; \\
\hline 178 & 2009 & Demetgul, M; Tansel, Ibrahim N; Taskin, S; \\
\hline 179 & 2009 & Alegre, Enrique; Alaiz-Rodriguez, Rocio; Barreiro, Joaquín; Ruiz, Jonathan; \\
\hline 180 & 2009 & Munguía, J; Ciurana, J; Riba, C; \\
\hline 181 & 2008 & Caydaş, Ulaş; Hascalık, Ahmet; \\
\hline 182 & 2008 & Yuan, Jin; Wang, Kesheng; Yu, Tao; Fang, Minglun; \\
\hline 183 & 2008 & Wang, Chih-Hsuan; \\
\hline 184 & 2008 & Khan, Aftab A; Moyne, James R; Tilbury, Dawn M; \\
\hline 185 & 2008 & Jager, Mark; Knoll, Christian; Hamprecht, Fred A; \\
\hline 186 & 2008 & Tsai, Du-Ming; Lai, Shia-Chih; \\
\hline 187 & 2008 & Bellini, Alberto; Filippetti, Fiorenzo; Tassoni, Carla; Capolino, Gérard-André; \\
\hline 188 & 2008 & Lei, Yaguo; He, Zhengijia; Zi, Yanyang; \\
\hline
\end{tabular}

TITLE

Diagnosis of machining outcomes based on machine learning with Logical Analysis of Data omparative study of three evolutionary algorithms coupled with neural network model for optimization of electric discharge machining process parameters Gearbox fault identification and classification with convolutional neural networks Nonlinear Gaussian Belief Network based fault diagnosis for industrial processes

Rolling bearing fault diagnosis using an optimization deep belief network
Fult diagnosis in high-speed train running gears with improved deep belief networks

Multimodel deep support vector classification with homologous features and its application to gearbox fault diagnosis ong short term memory networks for anomaly detection in time series

A dynamic quality control approach by improving dominant factors based on improved principal component analysis ondition-based maintenance for medium speed diesel engines used in vessels in operation random-key encoded harmony search approach for energy-efficient troduction scheduling Artificial Intelligence Techniques Multi-objective optimization of parallel machine scheduling integrated with multi-resources preventive maintenance planning Topology optimization algorithms for additive manufacturing An intelligent machine monitoring in system for energy prediction using a Gaussian Processs regression ool condition monitoring using K-star algorithm Development of a smart machining system using self-optimizing control

An approach to fault diagnosis of reciprocating compressor valves using Teager-Kaiser energy operator and deep belief networks rectural health monitoring by using a sparse coding-based deep learning algorithm with wireless sensor networks

A framework for root cause detection of sub-batch processing system for semiconductor manufacturing big data analytics \begin{tabular}{|l|l} 
A framework for root cause detection of sub-batch processing system for sem \\
\hline Defect inspection system for steel wire rods produced by hot rolling process \\
\hline Cantilever snap assemblies failure detection using syms and the rchht
\end{tabular}

evelopment of an accelerometer-based surface roughness prediction system in turning operations using multiple regression techniques

sing multi-sensor data fusion for vibration fault diagnosis of rolling element bearings by accelerometer and load cel

sector optimization of laser solid freeform fabrication system using a hierarchical mutable smart bee-fuzzy inference system and hybrid NSGA-II/self-organizing map An ensemble approach of machine learning in evaluation of mechanical property of the rapid prototyping fabricated prototype A flexible algorithm for fault diagnosis in a centrifugal pump with corrupted data and noise based on ANN and support vector machine with hyper-parameters optimization Robustness of thermal error compensation modeling models of CNC machine tools

ybrid GR-SVM for prediction of surface roughness in abrasive water jet machin

fillure diagnosis using deep belief learning based health state classification

emiconductor fault detection and classification for yield enhancement and manufacturing intelligence

(a) earning

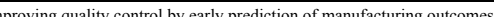

eel defect classification with max pooling on moltional ne atcomes

Automated surface inspection of cold-formed micro-parts

nhanced piecewise least squares approach for diagnosis of ill-conditioned multistation assembly with compliant parts

arameters optimization of advanced machining processeses using TLBO algorithm

Tynosis of defect propagation based on recurrent new

Directional textures auto-insspection using principal component analysis

Prectional textures auto-inspection using discrete cosine transform

rediction of surface roughness in CNC end milling by machine vision system using artificial neural network based on $2 \mathrm{D}$ Fourier transform

A neural network-based build time estimator for layer manufactured objects
ault detection and diagnosis of an industrial steam turbine using fusion of SVM (support vector machine) and ANFIS (adaptive neuro-fuzzy inference system) classifiers Theritificial neural network and genetic algorithms

Incipient gear box fault diagnosis using discrete wavelet transform (DWT) for feature extraction and classification using artificial neural network (ANN)

Restoration of blurred images for surface roughness evaluation using machine vision

he prediction of the building precision in the Laser Engineered Net Shaping process using advanced networks

virtual metrology system for semiconductor manufacturing

ault diagnosis of pneumatic systems with artificial neural network algorithm

se of contour signatures and classification methods to optimize the tool life in metal machining

A study on surface roughness in abrasive waterjet machining process using artifificial neural networks and regression analysis method

Reliable multi-objective optimization of high-speed WEDM process based on Gaussian process regression

Vecognition of semiconductor defect patterns using spatial filtering and spectral clustering

Defect detection in periodically patterned surfaces using independent tomine

Avances in diagnostic techniques for induction machines 


\begin{tabular}{|c|c|c|}
\hline ID & YEAR & AUTHOR \\
\hline 189 & 2008 & Alegre, Enrique; Barreiro, Joaquin; Castejón, Manuel; \\
\hline 190 & 2008 & Barreiro, J; Castejón, M; Alegre, E; Hernández, LK; \\
\hline 191 & 2007 & Widodo, Achmad; Yang, Bo-Suk; \\
\hline 192 & 2007 & Chien, Chen-Fu; Wang, Wen-Chih; Cheng, Jen-Chieh; \\
\hline 193 & 2007 & Zhang, Julie Z; Chen, Joseph C; \\
\hline 194 & 2007 & Ghosh, N; Ravi, YB; Patra, A; Mukhopadhyay, S; Paul, S; Mohanty, AR; Chattopadhyay, AB; \\
\hline 195 & 2007 & Shi, Dongfeng; Gindy, Nabil N; \\
\hline 196 & 2007 & Çolak, Oğuz; Kurbanoğlu, Cahit; Kayacan, M Cengiz; \\
\hline 197 & 2007 & Castejón, M; Alegre, E; Barreiro, J; Hernández, LK; \\
\hline 198 & 2006 & Chiang, Ko-Ta; Chang, Fu-Ping; \\
\hline 199 & 2006 & Dong, Jianfei; Subrahmanyam, KVR; San Wong, Yoke; Hong, Geok Soon; Mohanty, AR; \\
\hline 200 & 2006 & Abburi, NR; Dixit, US; \\
\hline 201 & 2006 & AbellánNebot, José Vcte; Morales-Menéndez, Rubén; Guevara, Antonio J Vallejo; Rodriguez, A Ciro; \\
\hline 202 & 2006 & Akbari, Ali Akbar; Fard, Amin Milani; Chegini, Amir Goodarzvand; \\
\hline 203 & 2006 & Kassim, Ashraf A; Mian, Zhu; Mannan, MA; \\
\hline 204 & 2006 & Zhang, Xiang; Krewet, Carsten; Kuhlenkötter, Bernd; \\
\hline 205 & 2005 & Riberiro, Bernardete; \\
\hline 206 & 2005 & Cho, Sohyung; Asfour, Shihab; Onar, Arzu; Kaundinya, Nandita; \\
\hline 207 & 2005 & Shin, Hyun Joon; Eom, Dong-Hwan; Kim, Sung-Shick; \\
\hline 208 & 2005 & Dey, Story; Stori, JA; \\
\hline 209 & 2005 & Vallejo, Antonio G; Nolazco-Flores, Juan A; Morales-Menéndez, Rubén; Sucar, L Enrique; Rodríguez, Ciro A; \\
\hline 210 & 2005 & Kohli, A; Dixit, US; \\
\hline 211 & 2005 & Lee, Kuang-Chyi; Ho, Shinn-Jang; Ho, Shinn-Ying; \\
\hline 212 & 2005 & Stemmer, M., Pavim, A., Adur, M., Deschamps, F., Schmitt, R., Hermes, R. \\
\hline 213 & 2004 & Sun, J; Rahman, M; Wong, YS; Hong, GS; \\
\hline 214 & 2004 & Haber, Rodolfo E; Jiménez, Jose E; Peres, C Ronei; Alique, José R; \\
\hline 215 & 2004 & Brezocnik, Miran; Kovacic, Miha; Ficko, Mirko; \\
\hline 216 & 2004 & Kassim, Ashraf A; Mian, Zhu; Mannan, MA; \\
\hline 217 & 2004 & Lee, BY; Yu, SF; Juan, H; \\
\hline 218 & 2003 & Ertekin, Yalcin M; Kwon, Yongjin; Tseng, Tzu-Liang Bill; \\
\hline 219 & 2003 & Choudhury, SK; Bartarya, G; \\
\hline 220 & 2003 & Risbood, KA; Dixit, US; Sahasrabudhe, AD; \\
\hline 221 & 2003 & Dweiri, F; Al-Jarrah, M; Al-Wedyan, H; \\
\hline 222 & 2003 & Lo, Ship-Peng; \\
\hline 223 & 2003 & Al-Najjar, Basim; Alsyouf, Imad; \\
\hline 224 & 2002 & Tosun, Nihat; Özler, Latif; \\
\hline 225 & 2002 & Benardos, PG; Vosniakos, G Cl; \\
\hline 226 & 2002 & Dimla, Dimla Eric; \\
\hline 227 & 2002 & Wang, Litao; Mehrabi, Mostafa G; Kannatey-Asibu Jr, Elijah; \\
\hline 228 & 2002 & Ho, Shinn-Ying; Lee, Kuang-Chyi; Chen, Shih-Shin; Ho, Shinn-Jang; \\
\hline 229 & 2002 & Kassim, Ashraf A; Mian, Zhu; Mannan, MA; \\
\hline 230 & 2001 & Abouelatta, OB; Madl, J; \\
\hline 231 & 2001 & Lee, BY; Tarng, YS; \\
\hline 232 & 2001 & Prasad, K Niranjan; Ramamoorthy, B; \\
\hline 233 & 2000 & Chen, Shang-Liang; Jen, YW; \\
\hline 234 & 2000 & El Ouafi, Abderrazak; Guillot, Michel; Bedrouni, Abdellah; \\
\hline 235 & 2000 & Al-Habaibeh, A; Gindy, N; \\
\hline 236 & 2000 & Li, Xiaoli; Dong, Shen; Venuvinod, PK; \\
\hline 237 & 2000 & Jack LB, Nandi AK \\
\hline 238 & 2000 & Mannan, MA; Kassim, Ashraf A; Jing, Ma; \\
\hline 239 & 1999 & Tsai, Yu-Hsuan; Chen, Joseph C; Lou, Shi-Jer; \\
\hline 240 & 1999 & Li, Xiaoli; Dong, Shen; Yuan, Zhejun; \\
\hline 241 & 1999 & Kuo, RJ; Cohen, PH; \\
\hline 242 & 1998 & Niu, YM; Wong, YS; Hong, GS; \\
\hline 243 & 1998 & Tsai, Du-Ming; Chen, Jeng-Jong; Chen, Jeng-Fung; \\
\hline 244 & 1997 & Bahr, B; Motavalli, S; Arfi, T; \\
\hline 245 & 1997 & Azouzi, R; Guillot, M; \\
\hline 246 & 1997 & Xiaoli, Li; Yingxue, Yao; Zhejun, Yuan; \\
\hline 247 & 1997 & Karthik, A; Chandra, S; Ramamoorthy, B; Das, S; \\
\hline 248 & 1996 & Jedrzzejewski, J; Kwaśny, W; \\
\hline 249 & 1995 & SI, Yoo; TJ, Ko; Kim, HS; \\
\hline 250 & 1991 & Hoy, DEP; Yu, F; \\
\hline
\end{tabular}

TIILE

omputer vision and classification techniques on the surface finish control in machining processeses

Support vector rarschine in machinenc condition monitoring and faut diagnosis

ata mining for yeide enhancement ti semiconductor manufucturing and an empirical study

The development of a nin-process surface roughness adaptive control system in end milling operations

Stimation of tool wear during CNC milling using neural network-based sensor fusien

lool wear predictive model based on least squares support vector machines
Villing surface roughness prediction using evolutionary
programming methods

Mng surface roughness predclon ning evolutionary programming meth

pptimization of the WEDM process of particle-reinforced material with multiple performance characteristics using grey relational analysis

ayssian-inference-based neural networks for tool wear stimation

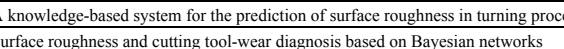

An effective image based surface roughness sstimation approach using neural network

Tool condition classification using Hidden Markov Model based on fractal analysis of machined surface textures

Automatic classification of defects on the product surface in grinding and polishing

Support vector machines for quality monitoring in a plastici injection molding process

Tool breakage detection using support vector machine learning in a milling procecss

One-class support vector machines-an application in machine fault detection

A Bayesian network approach to root cause diagnosis of process valt

A neural-network-based methodology for the prediction of surface roughness in a turning process

Acurate estimation of surface roughness from texture features of the surface image using an adaphive neuro-fizzy inference system

ullticlassification of tool wear with support vector machine by manufiacturing loss consideration

In investigation of tool-wear monitoring in a high-speed machining process

rediction of surface roughness with genetic programming

Conncectivity oriented fast Hough transform for tool wear monitoring

lentification of common sensory features for the control of $\mathrm{CNC}$ milling operations under varying cutting conditions

Role of temperature and surface finish in predicting tool wear using neural network and design of experiments

Prediction of surface roughness and dimensional deviation by measuring cutting forces and vibrations in turning process

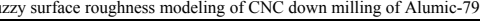

In adaptive-network based fuzzy inferencese system for prediction of workpicec surface roughness in end milling

Selecting the most ffficient maintenance approach using fiuzzy multiple criteria deceision making

Prediction of surface roughness in CNC face milling using neural networks and Taguchisis desegn of experiments

The corcelation of vibration signal featurues to cutting tool wear in a metal turning operation

Hidden Markov model-based tool wear monitoring in turning

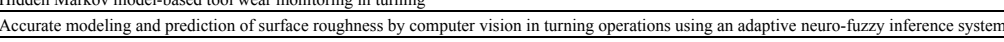

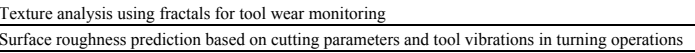

Surface roughness inspection by computer vision in turning operations

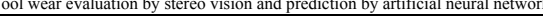

Data fusion neural network for tool condition monitoring in CNC milling machining

Iccuracy enhance ement of multi-axis CNC machines through on-line neurocompensation

new approach for systematic design of condition monitoring yystems for milling processeses

Hybrid learning for tool wear monitoring

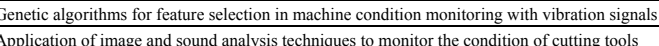

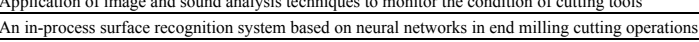

Discrete wavelet trans form for too b breakagae monitioring

Multisensor integration for on-line tool wear estimation through radial basis fiuction networks and fiuzy neural networl

nn intelligent sensor system approach for reliable tool flank wear recognition

Avision system for surface roughness assessment using
Sensor fusion for monitoring machine tool condition

On-line predicition of surfice finish and dimensional deviation in turning using neural network based sensor fusion

On-line tool condition monitoring system with wavelet fuzzy neural networl

sD tool wear measurement and visualisation using stereo imaging

Artificial intelligence tools in diagnostics of machine tool drives
Tool wear monitoring in nilling operation nuing ART2 neural netrind

Tool wear monitoring in milling operation using ART 2 neurl
Surface quality assessment using computer vision methods 